\title{
Método Combinado Análisis de Conglomerado y Análisis Discriminante Multivariado para Identificar y Evaluar Perfiles de Eficiencia Financiera en Empresas Exportadoras
}

\author{
Tomás J. Fontalvo, Efraín J. De La Hoz y José Morelos \\ Universidad de Cartagena, Facultad de Ciencias Económicas, Programa Administración Industrial. Campus \\ Piedra de Bolívar, Cartagena-Colombia. (e-mail: tfontalvoh@unicartagena.edu.co; \\ edelahozg@unicartagena.edu.co; jmorelosg@unicartagena.edu.co).
}

Recibido Dic. 5, 2017; Aceptado Mar. 6, 2018; Versión final Abr. 17, 2018, Publicado Oct. 2018

\section{Resumen}

Se presenta un método para definir y evaluar los perfiles de eficiencia financieros. Como fundamentación teórica se utilizó el cálculo multivariado y sus herramientas de Análisis de Conglomerado y Análisis Discriminante Multivariado ADM, también se utilizó Análisis Envolvente de Datos DEA con optimización en las salidas. Para esto se realizó una investigación con un enfoque racional y empírico, considerando 58 empresas medianas del sector exportador de la ciudad de Barranquilla-Colombia. Como resultado se aportó un método para establecer y evaluar perfiles de eficiencia financiera en las empresas exportadoras medianas de la zona. Se determinan también los perfiles de eficiencia financiera del sector exportador. Los niveles de eficiencia de los perfiles financieros de los clúster fueron $41,17 \%, 53,82 \%$ y $54,01 \%$ respectivamente. Se concluye que el análisis de conglomerados mostró condiciones favorables para clasificar grupos empresariales con perfiles de eficiencia financiera diferenciada.

\section{Combined Method of Conglomerate Analysis and Multivariate Discriminant Analysis to Identify and Evaluate Financial Efficiency Profiles in Exporting Companies}

\begin{abstract}
A method to define and evaluate financial efficiency profiles is presented. The multivariate calculus and its tools of Conglomerate Analysis and Multivariate Discriminant Analysis ADM were used as a theoretical basis. Data Envelopment Analysis (DEA) with output optimization was also used. For this, a rational and empirical approach research was done, considering 58 medium-sized companies of the export sector of the city of Barranquilla in Colombia. As a result, a method was provided to establish and evaluate financial efficiency profiles in medium-sized export companies of the area. The financial efficiency profiles of the export sector are also determined. The efficiency levels of the financial profiles of the clusters were $41.17 \%, 53.82 \%$ and $54.01 \%$ respectively. It is concluded that the cluster analysis showed favorable conditions to classify business groups with profiles of differentiated financial efficiency.
\end{abstract}

Keywords: financial efficiency profiles, conglomerate analysis, discriminant analysis, data envelopment analysis 


\section{INTRODUCCIÓN}

La globalización de la economía ha generado contextos de competitividad mucho más exigentes, dentro de este nuevo orden de competencia mundial, las empresas exportadoras juegan un papel determinante para generar competencia a nivel regional, nacional e internacional. Barranquilla-Colombia, actualmente es una ciudad con vocación exportadora, por las diferentes zonas francas e inversiones a nivel de logística portuaria y terrestre. Desde esta perspectiva hoy cobra importancia el análisis y estudio de los niveles de eficiencia de los diferentes clúster exportadores de la ciudad de Barranquilla. Lo anterior constituye un espacio de investigación para determinar cómo están desarrolladas las empresas exportadoras medianas de la ciudad de Barranquilla y cual son los niveles de eficiencia financiera de los grupos empresariales para responder a las condiciones de competencia y globalización de estas.

De lo anterior en esta investigación surgen las siguientes preguntas problema. ¿Cuáles son los rubros financieros que permiten calcular la eficiencia del sector exportador mediano de la ciudad de Barranquilla?, ¿Por medio de que técnicas de cálculo multivariado se pueden calcular perfiles o clúster?, ¿Cómo calcular los perfiles financieros de las empresas medianas exportadoras de la ciudad de Barranquilla?, ¿Cómo valorar la consistencia de agrupamiento de los perfiles empresariales?, ¿Cómo determinar los niveles de madurez y desarrollo de los perfiles financieros de las empresas medianas del sector exportador?, ¿Cómo determinar los perfiles de eficiencia financiera de las empresas medianas exportadoras de la ciudad de Barranquilla? ¿Cómo determinar los Ranking de eficiencia de los perfiles financieros empresariales de las empresas exportadoras medianas de la ciudad de Barranquilla Colombia?

En concordancia con lo anterior otras investigaciones han trabajado en la definición de perfiles o clúster de empresas exportadoras como lo señalan diferentes autores (De la Hoz, et al., 2016). También García et al, (2009) han utilizado el cálculo multivariado, específicamente el análisis de conglomerado en la pequeña y mediana empresa para analizar comportamiento de los clúster empresariales. Por lo anterior en esta investigación se tiene como intencionalidad generar valor al aportar un método que permita identificar y evaluar perfiles de eficiencia financiera de las empresas medianas del sector exportador de la ciudad de Barranquilla, con los cuales se pueden determinar el nivel de desarrollo de los grupos empresariales, así como los niveles de desempeño en términos de eficiencia de tal forma que se tengan criterios claros para el análisis y toma de decisiones en estos clúster empresariales y se puedan tomar decisiones que contribuyan con la competitividad y posicionamiento de las empresas medianas exportadoras de la ciudad de Barranquilla Colombia

Así mismo, es importante señalar que autores como: Aznar et al., (2011aㅡ); Aznar et al., (2011b); De la Hoz et al., (2014); García et al., (2011); Yujie y Hsuanshih, (2008), han considerado metodologías variables y rubros financieros similares a los de esta investigación para analizar sectores empresariales. Vera y Ganga (2007) desarrollaron toda una fundamentación conceptual y desarrollo teórico asociados con la conformación de los clúster empresariales, específicamente analizan las características de su composición y los factores determinantes para su desarrollo. En este mismo sentido Gómez (2017), también plantea la importancia de identificar una serie de factores fundamentales en la conformación de los clúster empresariales y señala como variables significativas la cooperación, redes sociales, conocimiento e innovación, lo cual incide en la competitividad del sector. Por otro lado Rodríguez, et al., (2013) han estudiado la importancia de las redes o clúster empresariales y su incidencia en la innovación en las empresas de Colombia. Manzaneque, et al., (2010) en su investigación utilizan herramientas estadísticas para analizar los clúster y su incidencia en la competitividad de los grupos empresariales.

El análisis de conglomerados o de cluster identifica patrones en una muestra de observaciones para clasificarla en grupos homogéneos o con características similares entre ellos (Peña, 2002). Se utiliza para construcciones de estructuras jerárquicas, partición de observaciones en un número de grupo definido y reducción de la dimensión o número de variables de un problema analizado (Peña, 2002). En la clusterización de las observaciones, se utiliza un análisis de proximidad para evaluar el nivel similaridad o disimilaridad de las observaciones y criterios de eslabonamiento o agrupamiento. La similaridad, mide que tan parecidas son las muestras. En el caso de clasificar variables se utiliza frecuentemente el coeficiente de Pearson cuadrado siendo $R_{i j}$ la medida de correlación de Pearson entre la muestra $i$ y la muestra $j$, con $X_{i k}$ valor de la muestra $i$ en la variable $k, X_{j k}$ valor de la muestra $j$ en la variable $k, r$ número total de variables y $\bar{X}_{k}$ promedio de la variable $k$ en todas las muestras, ver ecuación 1 (De la Garza, et al., 2013).

$$
R_{i j}^{2}=\frac{\sum_{k=1}^{r}\left(X_{i k}-\bar{X}_{k}\right)\left(X_{j k}-\bar{X}_{k}\right)}{\left[\sum_{k=1}^{r}\left(X_{i k}-\bar{X}_{k}\right)^{2} \sum_{k=1}^{r}\left(X_{j k}-\bar{X}_{k}\right)^{2}\right]^{2}}
$$


Por su parte, la disimilaridad establece que tan diferente que son las muestras. La medida más utilizada es la distancia euclídea al cuadrado donde $d^{2}{ }_{i j}$ representa la distancia entre la muestra $i$ y la muestra $j$ calculada mediante la ecuación 2, con $r$ es el número total de variables, $X_{i k}$ el valor de la muestra $i$ en la variable $k$ y $X_{j k}$ el valor de la muestra $j$ en la variable $k$.(De la Garza et al., 2013).

$$
d^{2}{ }_{i j}=\sum_{k=1}^{r}\left(X_{i k}-X_{j k}\right)^{2}
$$

En el proceso de eslabonamiento se conforman los grupos o cluster según el nivel de parecido de las observaciones, de manera que conformen grupos homogéneos (De la Garza et al., 2013). En este propósito, el método de la varianza mínima o método de Ward se caracteriza por minimizar el incremento de la varianza en la conformación de conglomerados de manera de lograr grupos con buen nivel de homogeneidad (Catena, et al., 2003). Se mide mediante la suma de cuadrados $W$, con $X_{i g}$ valor de la variable del individuo $i$ en el grupo $g, \overline{X_{g}}$ valor promedio del grupo $g, n_{g}$ el número de individuos del grupo $g, G$ el número total de grupos, ver ecuación 3. Para elegir los grupos a unirse se utiliza la ecuación 4 , con $n_{a}$ y $n_{b}$ el número de individuos de cada grupo (a y b) a agruparse, $\overline{X_{a}}$ y $\overline{X_{b}}$ valor medio de los grupo a y b respectivamente. (Peña, 2002):

$$
\begin{aligned}
& W=\sum_{g=1}^{G} \sum_{i=1}^{n_{g}}\left(X_{i g}-\overline{X_{g}}\right)\left(X_{i g}-\overline{X_{g}}\right)^{\prime} \\
& \min \frac{n_{a} n_{b}}{n_{a}+n_{b}}\left(\overline{X_{a}}-\overline{X_{b}}\right)^{\prime}\left(\overline{X_{a}}-\overline{X_{b}}\right)
\end{aligned}
$$

Por otra parte, el Análisis Discriminante Multivariado ADM es una técnica de clasificación supervisada (Lai et al., 2013; Yu y Zhao, 2018). Utiliza observaciones pertenecientes a clases o grupos para generar funciones discriminantes, capaces de clasificarlas en clases específicas de manera óptima, es utilizada para reducir las variables en problemas que contienen un gran número de variables, datos u observaciones, los costos de recolección y la velocidad de procesamiento (Glen, 2001; Iduseri y Osemwenkhae, 2015). Sea $\vec{X}_{=}\left(\mathrm{X}_{1}, \mathrm{X}_{2}, \ldots\right.$, $\mathrm{X}_{p}$ ) un vector con $p$ variables aleatorias del problema, entonces se define $Y_{i}$ la función discriminante a partir de la combinación lineal de variables originales $X_{1}, X_{2}, \ldots X_{p}$, de manera que se minimice la variación intra grupal y se maximice la variación entre grupo. Ver ecuación 5 , con $\beta_{i 0}$ una constante, $\beta_{i j}$ ponderación discriminante de la variable independiente $X_{j}, j=1, \ldots, p$.

$$
Y_{i}=\beta_{i 0}+\beta_{i 1} X_{1}+\cdots+\beta_{i p} X p, \mathrm{i}=1, \ldots, \min \{\mathrm{p}, \mathrm{g}-1\}
$$

De igual manera investigaciones desarrolladas por Gómez, et al., (2017) en sectores empresariales también se apoyan en la técnica de ADM para establecer el nivel de confianza de pertenencia o discriminación de grupos empresariales al igual que se realiza en esta investigación.

El Análisis Envolvente de Datos (DEA) es una técnica no paramétrica que utiliza la programación lineal para calcular la eficiencia entre un conjunto de unidades de toma de decisión (DMU: Decisión Making Units) a partir de datos de entrada y salida (Ehrgott et al., 2018). Autores como: Falavigna et al., (2018); Galadera et al., (2018); Ghiyasi, (2017); Niknazar y Bourgault, (2017) y Zhang et al., (2017), han considerado DEA para evaluar diversos contextos organizacionales para evaluar su eficiencia lo que muestra su importancia analítica. En esta investigación se articulan los planteamientos asociados al análisis de clúster, ADM junto con la técnica de análisis envolvente de datos DEA para proponer un método en el que se establecen y evalúan perfiles de eficiencia financiera en las empresas exportadoras medianas de la ciudad de Barranquilla.

\section{METODLOGİA}

Para el desarrollo del conocimiento se utilizó un análisis racional empírico, con el fin de definir y estructurar las variables del modelo y los criterios de clusterización, discriminación y eficiencia del método ConglomeradoADM-DEA para definir los perfiles de eficiencia financiera de las empresas medianas del sector exportador objeto de esta investigación.

Como método de desarrollo de esta investigación, se partió de las diferentes actividades que se presentan en la Figura 1. Para lo anterior se partió de los estados financieros de un grupo empresarial exportador de 58 empresas medianas del sector exportador de Barranquilla-Colombia. Para esto se utilizó información primaria tomada del SIREM (Sistema de Información y Reporte Empresarial). Con los rubros y variables seleccionadas para el modelo DEA se aplicó la técnica de cálculo multivariado análisis conglomerado para la identificación de los perfiles financieros de las empresas objeto de esta investigación para lo cual se trabajó con el indicador estadístico de varianza Ward como criterio de agrupamiento y con la distancia euclídea como medida de 
similitud. Seguidamente se estableció el nivel de confianza de la clasificación de los perfiles financieros por medio de ADM para analizar el nivel de discriminación de los clúster empresariales financieros. Con la información estadística generada a través de la aplicación de ADM se estableció el nivel de madurez y desarrollo del os perfiles empresariales financieros de las empresas exportadoras de la ciudad de Barranquilla-Colombia. Finalmente se establecieron los perfiles de eficiencia financiera de las empresas medianas del sector exportador de la ciudad de Barranquilla Colombia, por medio de la técnica DEA con lo cual se determinó la eficiencia de los grupos empresariales. Lo anterior permitió establecer rankings de eficiencia financiera de los perfiles empresariales identificados en el análisis de conglomerado.

Para la aplicación de las herramientas de cálculo multivariado se utilizó el software Minitab 16 y para el cálculo de análisis envolvente de datos DEA se utilizó el software DEA Solver Pro.

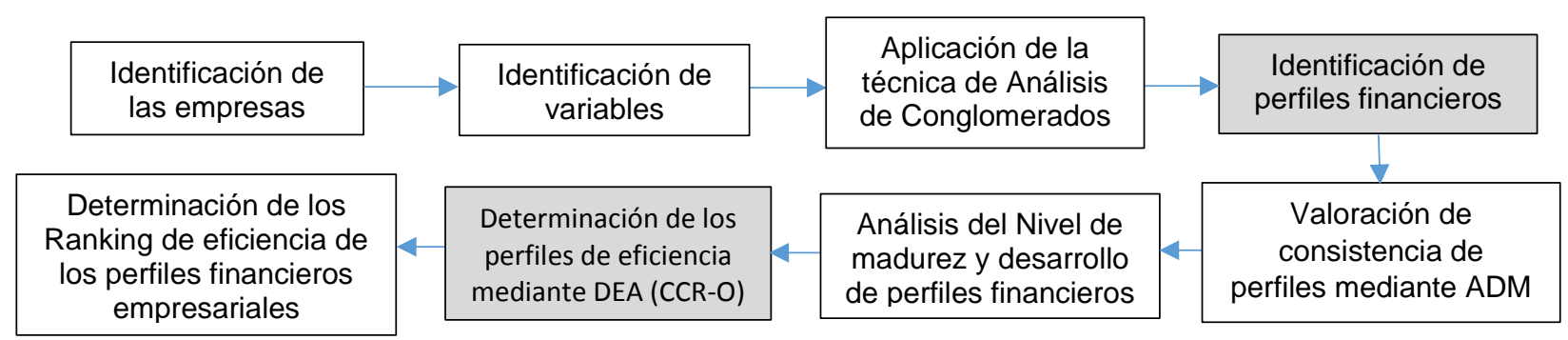

Fig. 1: Método para definir perfiles financieros de eficiencia empresariales

\section{RESULTADOS Y DISCUSIÒN}

Para el desarrollo de los perfiles inicialmente se partió de los estados financieros de las empresas medianas exportadora de la ciudad de Barranquilla. Se tomaron como variables para aplicar la técnica de análisis de conglomerado los rubros financieros de entrada Total Activo, Patrimonio y Total pasivos y como rubros financieros de salida se utilizaron como Utilidad neta y Utilidad operacional. Como resultado del análisis de conglomerado, en la Tabla 1 se muestran las distancias promedios y máxima desde el centroide de cada grupo de conglomerado, observando una distancia promedio desde el centroide de 2,50E+09 mucho menor que la distancia máxima desde el centroide de 7,17E+09 para el perfil 1, lo que evidencia el alto nivel de homogeneidad de los grupos identificados por el análisis de conglomerados. Es importante señalar que los conglomerados 1,2 y 3 explicitan los perfiles de financieros empresariales 1,2 y 3 respectivamente.

Tabla 1: Distancia promedio y máxima desde centroide de los perfiles financieros

\begin{tabular}{|l|c|c|c|c|}
\hline & $\begin{array}{c}\text { Número de } \\
\text { observaciones }\end{array}$ & $\begin{array}{c}\text { Suma de } \\
\text { cuadrados del } \\
\text { conglomerado }\end{array}$ & $\begin{array}{c}\text { Promedio } \\
\text { desde el } \\
\text { centroide }\end{array}$ & $\begin{array}{c}\text { Distancia } \\
\text { máxima desde } \\
\text { centroide }\end{array}$ \\
\hline Perfil financiero 1 & 14 & $2,05 \mathrm{E}+20$ & $3,43 \mathrm{E}+09$ & $7,17 \mathrm{E}+09$ \\
\hline Perfil financiero 2 & 21 & $4,46 \mathrm{E}+19$ & $1,30 \mathrm{E}+09$ & $3,09 \mathrm{E}+09$ \\
\hline Perfil financiero 3 & 23 & $2,08 \mathrm{E}+20$ & $2,78 \mathrm{E}+09$ & $5,24 \mathrm{E}+09$ \\
\cline { 2 - 4 } & & Promedio & $2,50 \mathrm{E}+09$ & \multicolumn{2}{c}{} \\
\cline { 2 - 4 } & & & &
\end{tabular}

De igual manera, en la Tabla 2 se muestran las distancias entre centroides de conglomerados de donde se puede observar las diferencias entre los perfiles financieros de las empresas del sector exportador de Barranquilla Colombia correspondiente a 9,5E+09 (promedio de distancias entre centroides) mayor que la distancia promedio dentro del centroide $(2,50 \mathrm{E}+09)$.

Tabla 2: Distancia entre centroides de perfiles financieros

\begin{tabular}{|l|c|c|c|}
\cline { 2 - 4 } \multicolumn{1}{c|}{} & Perfil financiero 1 & Perfil financiero 2 & Perfil financiero 3 \\
\hline Perfil financiero 1 & $0,00 \mathrm{E}+00$ & $1,41 \mathrm{E}+10$ & $1,00 \mathrm{E}+10$ \\
\hline Perfil financiero 2 & $1,41 \mathrm{E}+10$ & $0,00 \mathrm{E}+00$ & $4 ., \mathrm{E}+09$ \\
\hline Perfil financiero 3 & $1,00 \mathrm{E}+10$ & $4,40 \mathrm{E}+09$ & $0,00 \mathrm{E}+00$ \\
\hline
\end{tabular}


Para el desarrollo del método propuesto se utilizaron distintos criterios de medidas de distancia como son la euclídea, euclídea cuadrada, manhattan, pearson y pearson cuadrada y de eslabonamiento o vinculación como son el promedio, centroide, completo, macquitty, mediana, individual y ward sin embargo en el proceso de análisis y estudio se priorizó el criterio de ward y la medida euclidiana que mostraron mejor poder de clasificación para determinar los tres clúster empresariales del sector exportador en la ciudad de Barranquilla.

De la aplicación de la técnica de análisis de conglomerado se puede validar la capacidad de clasificación de los rubros financieros para determinar los perfiles financieros empresariales, que permitan clasificar perfiles de eficiencia financiera para el sector, lo que posibilita analizar los desempeños de eficiencia de los grupos empresariales o clúster y a su vez tener criterios claros para tomar acciones sobre las variables que contribuyan a mejorar la competitividad de las empresas por perfiles. A continuación se presentan los criterios para validar y analizar la pertinencia de los perfiles financieros de las empresas medianas del sector de Barranquilla-Colombia, mediante la técnica de cálculo ADM.

Con el fin de analizar la variabilidad de los perfiles empresariales del sector de empresas exportadoras medianas de la ciudad de Barranquilla se utilizó la técnica ADM. En la Tabla 3 se presentan los niveles de clasificación correcta de los perfiles empresariales observándose $96.6 \%$ de clasificación total correcta que representa excelente capacidad de discriminación de los 3 perfiles definidos previamente atraves del análisis de conglomerado. Lo anterior muestra la homogeneidad de los perfiles empresariales financieros y la heterogeneidad entre los perfiles definidos previamente.

En este sentido esta investigación presenta resultados muy superiores a otros en donde la capacidad de discriminación alcanzó el 47,5\% (Fontalvo, et al., 2014)

Tabla 3: Nivel de clasificación correcta de perfiles financieros por ADM

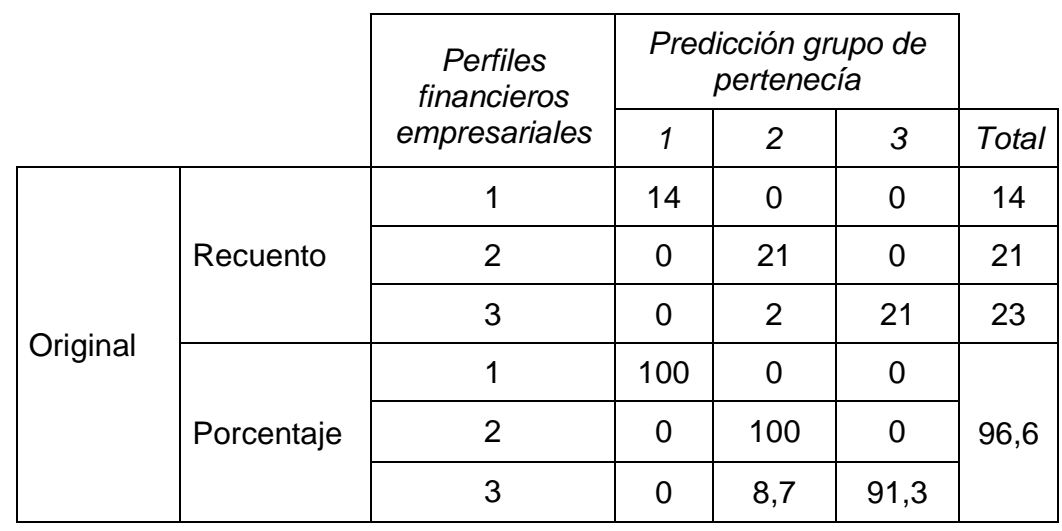

Con la aplicación de la técnica de ADM esta investigación prueba la capacidad de discriminación de los perfiles empresariales exportador de las empresas medianas de la ciudad de Barranquilla cuando se utiliza el análisis de conglomerado y se verifica la pertenencia o discriminación con ADM, a partir de los rubros Total Activos, Patrimonio, Total Pasivos, Utilidad Neta y Utilidad Operacional asociados a los estados financieros de las empresas objeto de esta investigación. Lo cual valida la capacidad y utilidad del método propuesto presentado en la Figura 1. De igual forma, del análisis de los perfiles financieros empresariales también se puede analizar qué nivel de madurez y desarrollo de los 3 sectores empresariales, cuándo se evalúan en términos del desempeño de los rubros financieros Total Activos, Patrimonio, Total Pasivos, Utilidad Neta y Utilidad Operacional. De la Tabla 4 se observa que el clúster que presenta mejor desempeño en términos de utilidad es el perfil empresarial financiero 1, seguido del perfil empresarial financiero 2 y 3 respectivamente.

Tabla 4: Media de los perfiles empresariales financieros

\begin{tabular}{|c|c|c|c|c|}
\cline { 3 - 5 } \multicolumn{2}{c}{} & \multicolumn{4}{c|}{ Medias de grupo } \\
\hline Variable & Media agrupada & 1 & 2 & 3 \\
\hline Total Activos & 8422206246 & $1,52 \mathrm{E}+10$ & $4,40 \mathrm{E}+09$ & $7,97 \mathrm{E}+09$ \\
\hline Patrimonio & 3004410429 & $3,93 \mathrm{E}+09$ & $1,82 \mathrm{E}+09$ & $3,52 \mathrm{E}+09$ \\
\hline Total Pasivos & 5368856579 & $1,13 \mathrm{E}+10$ & $2,53 \mathrm{E}+09$ & $4,37 \mathrm{E}+09$ \\
\hline Utilidad Neta & 492193470 & $8,46 \mathrm{E}+08$ & $1,78 \mathrm{E}+08$ & $5,63 \mathrm{E}+08$ \\
\hline Utilidad Operacional & 997519582 & $1,62 \mathrm{E}+09$ & $5,35 \mathrm{E}+08$ & $1,04 \mathrm{E}+09$ \\
\hline
\end{tabular}


La Tabla 5 muestra la homogeneidad de las variables, cuando se analizan dentro de los perfiles financieros. Se observan los perfiles 1 y 3 los más homogéneos, cuando se confrontan las deviaciones estándar promedio de la Tabla 5. Así mismo las variables utilidad operacional y utilidad neta representan son las que muestran mayor heterogeneidad entre perfiles financieros. Una vez definido los perfiles empresariales financieros del sector exportador en la ciudad de Barranquilla, se procedió a calcular los niveles de eficiencia de los 3 clúster empresariales mediante el análisis envolvente de datos DEA con un enfoque de optimización de las variables de salida. En la Tabla 6 se observan los niveles de eficiencia de los 3 perfiles financieros definidos en el análisis de conglomerado. Los resultados muestran el perfil empresarial 3 con mejor eficiencia financiera $54,011 \%$ seguido del perfil 2 con 53,82\% y finalmente grupo empresarial identificado con el perfil 1 obtuvo el nivel de eficiencia más bajo con $41,48 \%$. Lo que evidencia la optimización de las variables de entrada.

Una vez definido los perfiles empresariales financieros del sector exportador en la ciudad de Barranquilla, se procedió a calcular los niveles de eficiencia de los 3 clúster empresariales mediante el análisis envolvente de datos DEA con un enfoque de optimización de las variables de salida. En la Tabla 6 se observan los niveles de eficiencia de los 3 perfiles financieros definidos en el análisis de conglomerado. Los resultados muestran el perfil empresarial 3 con mejor eficiencia financiera $54,011 \%$ seguido del perfil 2 con $53,82 \%$ y finalmente grupo empresarial identificado con el perfil 1 obtuvo el nivel de eficiencia más bajo con $41,48 \%$. Lo que evidencia la optimización de las variables de entrada.

Tabla 5: Desviación estándar por perfiles empresariales financieros

\begin{tabular}{|c|c|c|c|c|}
\cline { 3 - 5 } \multicolumn{2}{c}{ Variable } & \multicolumn{3}{c|}{ Desviación estándar para grupo } \\
\hline & $\begin{array}{c}\text { Desviación estándar } \\
\text { agrupada }\end{array}$ & 1 & 2 & 3 \\
\hline Total Activos & $1,56 \mathrm{E}+09$ & $2,42 \mathrm{E}+09$ & $5,58 \mathrm{E}+08$ & $1,54 \mathrm{E}+09$ \\
\hline Patrimonio & $1,54 \mathrm{E}+09$ & $1,84 \mathrm{E}+09$ & $8,63 \mathrm{E}+08$ & $1,81 \mathrm{E}+09$ \\
\hline Total pasivos & $1,53 \mathrm{E}+09$ & $2,15 \mathrm{E}+09$ & $7,78 \mathrm{E}+08$ & $1,60 \mathrm{E}+09$ \\
\hline Utilidad Neta & $5,15 \mathrm{E}+08$ & $7,43 \mathrm{E}+08$ & $1,60 \mathrm{E}+08$ & $5,59 \mathrm{E}+08$ \\
\hline Utilidad operacional & $9,53 \mathrm{E}+08$ & $1,18 \mathrm{E}+09$ & $7,36 \mathrm{E}+08$ & $9,78 \mathrm{E}+08$ \\
\hline & Promedio & $1,67 \mathrm{E}+09$ & $6,19 \mathrm{E}+08$ & $1,30 \mathrm{E}+09$ \\
\hline
\end{tabular}

Tabla 6: Perfiles de eficiencia financiera de las empresas exportadoras mediante DEA (CCR-O)

\begin{tabular}{|c|c|}
\hline \multicolumn{2}{|c|}{ Perfil 1 } \\
\hline Número de DMU & 23 \\
\hline Eficiencia Promedio & 0,4178 \\
\hline Desviación estándar & 0,3445 \\
\hline Maximum & 1 \\
\hline Minimum & 0,0704 \\
\hline
\end{tabular}

\begin{tabular}{|c|c|}
\hline \multicolumn{2}{|c|}{ Perfil 2 } \\
\hline Número de DMU & 21 \\
\hline Eficiencia Promedio & 0,5382 \\
\hline Desviación estándar & 0,3357 \\
\hline Maximum & 1 \\
\hline Minimum & 0,0790 \\
\hline
\end{tabular}

\begin{tabular}{|c|c|}
\hline \multicolumn{2}{|c|}{ Perfil 3 } \\
\hline Número de DMU & 14 \\
\hline Eficiencia Promedio & 0,5401 \\
\hline Desviación estándar & 0,3195 \\
\hline Maximum & 1 \\
\hline Minimum & 0,0240 \\
\hline
\end{tabular}

Otras investigaciones que han trabajado la eficiencia con subtotal de inventario, total de los activos corriente, propiedad planta y equipo, proveedores y la utilidad operacional, muestran que el promedio de eficiencia del grupo de estudio fue de $56,62 \%$, es decir muestran un desempeño ligeramente superior al promedio alcanzado por los perfiles de eficiencia financiera de las empresas exportadoras medianas (Fontalvo, et al., 2015). Así mismo, en otros contextos empresariales como el cooperativo en España se lograron aplicaciones de DEA con resultados de eficiencia del 79,8\% y 89,7\%, Belmonte y Plaza, (2008) y Arzubi y Berbel, (2002) determinaron índices de eficiencia del $83 \%$ en el sector lechero de Buenos Aires, lo que permite evidenciar la necesidad de mejora del sector.

El análisis DEA, igualmente permite establecer un ranking de eficiencia financiera de las empresas por perfil identificando las empresas más eficientes. De la Tabla 7 se puede observar el perfil 1 con más empresas eficientes (con 5 empresas), seguido del perfil 2 con 4 empresas eficientes y por último el perfil 3 con 3 empresas eficientes. Sin embargo también se puede observar si analizamos las eficiencias por cuartiles que el perfil 1 presenta 10 empresas por debajo del segundo cuartil (50\%), el perfil empresarial 2 presenta 7 empresas y el tercer perfil 3 presenta 8 empresas por debajo de segundo cuartil. 
Tabla 7: Ranking de los perfiles de eficiencia financiera de las empresas exportadoras medianas de la ciudad de Barranquilla

\begin{tabular}{|c|c|c|}
\hline 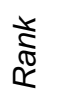 & $\begin{array}{c}\text { DMU del perfil de } \\
\text { eficiencia financiero } 1\end{array}$ & ఏั \\
\hline 1 & Interpelli s.a.s. & 1 \\
\hline 1 & Constructora rumie s.a.s & 1 \\
\hline 1 & Transnaval s.a.s. & 1 \\
\hline 1 & Boating international s.a. & 1 \\
\hline 5 & Ci metal trade sas & 0,809 \\
\hline 6 & Industrias sedal s.a. & 0,803 \\
\hline 7 & GGMC & 0,723 \\
\hline 8 & Colarquim s.a.s. & 0,463 \\
\hline 9 & Bolten limitada & 0,420 \\
\hline 10 & B/quilla ind. confecciones & 0,371 \\
\hline 11 & Latexcol s.a.s. & 0,296 \\
\hline 12 & Sedas del caribe s.a.s. & 0,275 \\
\hline 13 & Thermocoil Itda. & 0,241 \\
\hline 14 & Peleteria del oriente s.a. & 0,240 \\
\hline 15 & Frigal & 0,196 \\
\hline 16 & Aerosucre s. A. & 0,131 \\
\hline 17 & Venter colombia s.a.s. & 0,130 \\
\hline 18 & Oficaribe s.a.s & 0,103 \\
\hline 19 & Altamoda s.a.s. & 0,101 \\
\hline 20 & Inversiones trifer limitada & 0,094 \\
\hline 21 & Arquicentro del prado s.a. & 0,087 \\
\hline 22 & Pinillar limitada & 0,082 \\
\hline 23 & $\begin{array}{l}\text { Fabricación y montajes } \\
\text { industriales s.a. }\end{array}$ & 0,070 \\
\hline
\end{tabular}

\begin{tabular}{|c|c|c|}
\hline 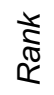 & $\begin{array}{c}D M U \text { del perfil de } \\
\text { eficiencia financiero } 2\end{array}$ & $\begin{array}{l}0 \\
\text { ठ̀ } \\
\text { c }\end{array}$ \\
\hline 1 & Syver s.a.s. & 1 \\
\hline 1 & Alutrafic led s.a.s. & 1 \\
\hline 1 & Prosigna s.a.s. & 1 \\
\hline 1 & I.b.s. zona franca s.a. & 1 \\
\hline 1 & Exotika leather s.a. & 1 \\
\hline 6 & Insumedical Itda. & 0,876 \\
\hline 7 & Productos Juliao & 0,823 \\
\hline 8 & Yesos y caolines s.a. & 0,711 \\
\hline 9 & Silicar limitada & 0,638 \\
\hline 10 & C. I. Pradaz Itda. & 0,520 \\
\hline 11 & Expopieles sas & 0,471 \\
\hline 12 & Rodko Itda & 0,410 \\
\hline 13 & Golden cute s.a.s & 0,312 \\
\hline 14 & Litoboston s.a.s. & 0,270 \\
\hline 15 & Intertrade s.a. & 0,257 \\
\hline 16 & C.I Aleados del cobre & 0,240 \\
\hline 17 & VSI s.a.s. & 0,225 \\
\hline 18 & Tempro s.a.s & 0,211 \\
\hline 19 & Barbatuscas s.a.s & 0,149 \\
\hline 20 & Enrique Roca s.a.s. & 0,11 \\
\hline 21 & Inversiones hagamos & 0,07 \\
\hline
\end{tabular}

\begin{tabular}{|c|c|c|}
\hline 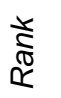 & $\begin{array}{c}D M U \text { del perfil de } \\
\text { eficiencia financiero } 3\end{array}$ & $\begin{array}{l}0 \\
\text { రু } \\
\text { ஸे }\end{array}$ \\
\hline 1 & Nova medica Itda & 1 \\
\hline 1 & Ccolandina c/cial s.a.s. & 1 \\
\hline 1 & Hotel B/quilla plaza s.a. & 1 \\
\hline 4 & Indutrade C/bia s.a.s. & 0,887 \\
\hline 5 & Clean energy Itda. & 0,672 \\
\hline 6 & Superbrix s.a. & 0,630 \\
\hline 7 & Hujueta s.a. & 0,462 \\
\hline 8 & Industria articueros s.a. & 0,457 \\
\hline 9 & Sonen int/nal s.a.s & 0,378 \\
\hline 10 & Thermotar Itda & 0,358 \\
\hline 11 & Ceramia sas & 0,305 \\
\hline 12 & Laboratorios best s.a. & 0,276 \\
\hline 13 & Suelos ingeniería s.a.s. & 0,109 \\
\hline 14 & Industrias yidi s.a. & 0,024 \\
\hline
\end{tabular}

\section{CONCLUSIONES}

Este trabajo de investigación aporta a la comunidad científica una metodología que articula las herramientas de cálculo multivariado: Análisis de conglomerado y Análisis discriminante y la herramienta de Análisis Envolvente de Datos, DEA para calcular eficiencia financiera de las empresas medianas exportadoras y valorar niveles de esta en grupos o perfiles empresariales. Con lo anterior, se establecieron criterios y variables necesarias para identificar los clúster empresariales financieros, los criterios para validar la conformación de estos clúster o perfiles y los niveles de eficiencia financiera de dichos perfiles empresariales. A partir de los resultados, se concluye que en el análisis de conglomerado los criterios de ward y la medida euclidiana mostraron poder de clasificación para determinar grupos empresariales homogéneos intragrupo y heterogéneos extragrupo. Lo anterior permitió desarrollar el modelo de ADM con 96.6\% de clasificación total correcta en el modelo predictivo del análisis discriminante para clasificar a futuro empresas en los perfiles de eficiencia analizados. Mediante DEA, se pudo establecer el ranking de los 3 perfiles de eficiencia financiera de las empresas medianas exportadoras de la ciudad de Barranquilla.

\section{REFERENCIAS}

Aznar, J., R. Cervelló-Royo y A. Romero, New Spanish Banking Conglomerates, Application of the Analytic Hierarchy Process (AHP) to their Market Value, International Research Journal of Finance and Economics, ISSN: 1450-2887, (78), 70-82 (2011 a)

Arzubi, A. y J. Berbel, Determinación de índices de eficiencia mediante DEA en explotaciones lecheras de Buenos Aires, Invest. Agr.: Prod. Sanid. Anim, ISSN: 0213-5035, 17(1), 103-123 (2002)

Aznar, J., R. Cervelló-Royo y F. García, Spanish Savings Banks and their Future Transformation into Private Capital 
Banks, Determining their Value by a Muticriteria Valuation Metodology, European Journal of Economics, Finance and Administrative Sciences, ISSN: 1450-2275, (35), 155-164 (2011 b)

Belmonte, L. y J. Plaza, Análisis de la eficiencia en las cooperativas de crédito en España, Una Propuesta metodológica basada en el análisis envolvente de datos (DEA). CIRIEC-España, doi.org/10.5209/rev_REVE.2013.v110.41445, Revista de Economía Pública, Social y Cooperativa, 63, 113- 133 (2008)

De La Hoz, E. y L. Lopez, Aplicación de Técnicas de Análisis de Conglomerado y Redes Neuronales Artificiales en la Evaluación del Potencial Exportador de una Empresa, doi: 10.4067/S071807642017000400009, Información Tecnológica, 28(4), 67-74 (2017)

De la Hoz, E., A. Gonzalez y A. Santana, Metodología de Medición del Potencial Exportador de las Organizaciones Empresariales, doi: 10.4067/S0718-07642016000600003, Información Tecnológica, 27(6), 11-18 (2016)

De la Hoz, E., H. Fontalvo y J. Morelos, Evaluación del Comportamiento de los Indicadores del Sector Petróleo y Gas en Colombia Mediante el Análisis Discriminnte, doi: 10.1016/S0186-1042(14)70159-7, Contaduría y administración 59(4), 167-191 (2014)

Ehrgott, M., A. Holder y O. Nohadani, Uncertain Data Envelopment Analysis, doi.org/10.1016/j.ejor.2018.01.005, European Journal of Operational Research, 268(1), 231-242 (2018)

Falavigna, G., R. Ippoliti y G. Ramello, DEA-based Malmquist productivity indexes for understanding courts reform, doi.org/10.1016/j.seps.2017.07.001, Socio-Economic Planning Sciences, (62), 31-43 (2018)

Fontalvo, T., A. Mendoza y D., Visbal, Análisis comparativo de eficiencia financiera: estudio de un caso del sector BASC en Barranquilla, doi.org/10.15665/rp.v13i2.483, Prospectiva, 13(2), 16-24 (2015)

Fontalvo, T., J. Morelos y E. De la Hoz, Aplicación del análisis discriminante para evaluar el mejoramiento de los indicadores financieros en las empresas del sector extracción de petróleo crudo y gas natural en Colombia, doi.org/10.4067/S0718-33052012000300006, Revista Soluciones de Postgrado EIA, 1(2), 1-16 (2014)

Galagedera, D., I. Roshdi y otros dos autores, A new network DEA model for mutual fund performance appraisal: An application to U.S. equity mutual funds, doi: 10.1016/j.omega.2017.06.006, Omega (United Kingdom), (77), 168-179 (2018)

García, A., J. Fernández y otros cinco autores, Análisis empírico de la influencia de la propiedad familiar sobre la orientación estratégica de las pequeñas y medianas empresas, doi.org/10.1016/S1135-2523(12)60077-X, Investigaciones, 15(1), 45-59 (2009)

García, F., F. Guijarro e I. Moya, Ranking Spanish Saving Banks: A Multicriteria Approach, doi: 10.1016/j.mcm.2010.02.015, Matematical and Computer Modelling, 52 (7-8), 1058-1065 (2011)

Ghiyasi, M., Efficiency Improvement and Resource Estimation: A Tradeoff Analysis, DOI: 10.1504/IJPQM.2018.10009555, International Journal of Productivity and Quality Management, en prensa, (2018)

Glen, J., Classification Accuracy in Discriminant Analysis: A Mixed Integer Programming Approach, doi: https://ezproxy.uninorte.edu.co:3099/10.1057/palgrave.jors.2601085, J. Oper. Res. Soc., 52(3), $328-339$ (2001)

Gómez, J., La importancia de los factores fundamentales en el clúster, doi: 10.15665/rde.v15i2.917, Dimensión Empresarial, 15(2), 141-153 (2017)

Iduseri, A. y J. Osemwenkhae, An Efficient Variable Selection Method for Predictive Discriminant Analysis, doi: 10.1007/s40745-015-0061-9, J.E. Ann. Data. Sci., 2(4), 489-504 (2015)

Lai, Z., Y. Xu y otros tres autores, Sparse Tensor Discriminant Analysis, doi.org/10.1109/TIP.2013.22646, IEEE Transactions on Image Processing, 22(10), 3904-3915 (2013)

Manzaneque, M., R. Banegas y D. García, Diferentes Procesos de Fracaso Empresarial, Un análisis dinámico a través de la aplicación de técnicas estadísticas clúster, Revista Europea de Dirección y Economía de la Empresa, 19(3), 67-88 (2010)

Morelos, J., T. Fontalvo y E. De La Hoz, Behaviour of Productivity Indicators and Financial Resources in the Field of Extraction and Exploitation of Minerals in Colombia, International Journal of Productivity and Quality Management, en prensa (2018)

Niknazar, P. y M. Bourgault, Analysis of Axioms and Assumptions of Data Envelopment Analysis (DEA), Application for Efficiency Measurement in Project Management Contexts, International Journal of Productivity and Quality Management, en prensa (2018)

Rodríguez, F., H. Gómez y J. Valencia, Redes empresariales locales, Investigación y desarrollo e innovación en la empresa, Clúster de herramientas de Caldas, Colombia, Estudios gerenciales, 29(127), 247-257 (2013)

Vera, J. y F. Ganga, Los clúster industriales: Precisión conceptual y desarrollo teórico, ISSN: 1900-7205, Cuadernos de Administración, 20(33), 303-322 (2007)

Yu, W. y C. Zhao, Sparse Exponential Discriminant Analysis and Its Application to Fault Diagnosis, doi: 10.1109/TIE.2017.2782232, IEEE Transactions on Industrial Electronics, 65(7), 5931-5940 (2018)

Yujie, W. y L. Hsuanshih, Clustering Method to Identify Representative Financial Ratios, doi: 10.1016/j.ins.2007.09.016, Information Sciences, 178(4), 1087-1097 (2008)

Zhang, Z., M. Wang y otros dos autores, Research on the Development Efficiency of Regional High-end Talent in China: A complex network approach, doi.org/10.1371/journal.pone.0188816, PLoS ONE, 12(12), 1-18 (2017) 